\title{
Waves in electric contact wires subjected to transversal impulses
}

\author{
Răzvan Andrei Oprea, Gabriel Popa* and Marius Alin Gheţi \\ University Politehnica of Bucharest, Department of Railway Vehicles, 313 Splaiul Independenţei, \\ Bucharest, Romania
}

\begin{abstract}
It is a well-known fact that the raising of the pantograph is followed by an electric arch. The reasons for this phenomenon are the oscillations of the pantograph and the oscillations of the contact line. Initially, these oscillations are not coupled. In this case, the mechanical contact between the two subsystems may be lost, and may generate electric arch. In the present work, the pantograph rising is modelled through a Dirac impulse. An analytic solution of the transverse waves is obtained by means of Fourier series. A phenomenological model of the wave attenuation is taken into account.
\end{abstract}

\section{Introduction}

Pantograph rising is followed by an electric arch which may alter current collection [1-5]. The electric arch follows to the mechanical contact oscillations. Initially, these oscillations are not coupled. Hence, the mechanical contact between the two subsystems may be lost, and electric arch may occur.

The present work is a study of the taut wire oscillations while the contact with the pantograph is lost.

The phenomena related to pantograph-wire contact can provide an optimized coupling mechanism in order to avoid the transitory phase of the power circuit connection or even the system damage. In order to achieve good high speed current collection, the contact wire geometry is kept within well-defined limits throughout the length of the overhead line.

Usually, this is obtained by supporting the contact wire from above with a second wire, known as the messenger wire or catenary.

This wire is allowed to follow the natural path of a wire strung between two points, which is known as a catenary curve, thus the use of catenary to describe this wire or sometimes the whole system.

The messenger wire is attached to the contact wire at regular intervals by vertical wires known as droppers or drop wires.

The contact wire tension will be about $20 \mathrm{kN}$ and the wave propagation speeds are between 382 and $572 \mathrm{~km} / \mathrm{h}$. The wire length is usually between 1000 and 1500 meters long, depending on the temperature ranges [6].

\footnotetext{
*Corresponding author: gabi21popa@yahoo.com
} 
Various models have been carried out in accordance with the purpose of the study. We shall remind several complex models which take into account all the essential parameters.

When modelling with the aid of the finite-element method, the contact line equipment is subdivided into differential elements which are linked by coupling mechanisms easily described in mathematical terms [6]. Such dynamic processes are described by systems of thousands of ordinary differential equations.

Analytical solution in the frequency area is based on a contact line system of infinite length, supported by jointed supports at finite spacing [7]. Each section may oscillate independently and the system is described by the Lagrange's equations [8]. An approximate solution may be obtained by means of Ritz method [9].

In order to reduce the order of the matrices needed in the analysis of the higher frequency processes, a suitable method is the use of frequency dependent finite elements [10]. This requires the calculation of the natural frequencies and of the natural vectors.

The reflected waves which propagate along the contact wire may be easily taken into account using d'Alembert wave equation [11]. The motion of the catenary elements is obtained from the superposition of the individual waves.

In the present study, the contact wire will be treated as a taut string between the droppers. An analytical expression is deduced for the waveform and amplitude.

\section{Transverse waves produced by Dirac impulses}

The transverse waves will be approached considering that when the pantograph raises it strikes the contact wire and it loses contact with the strip collector. In consequence, the first phase of the contact line movement may be regarded as a free oscillation of a taut wire.

The initial conditions are zero except for the contact point where we shall presume that the initial velocity is given by a Dirac impulse function.

The wire movement will be studied in the vertical plane only and, therefore, it is described by the one-dimensional wave equation [12]:

$$
\frac{\partial^{2} z}{\partial x^{2}}=\frac{1}{c^{2}} \frac{\partial^{2} z}{\partial t^{2}} .
$$

The horizontal axe is denoted by $x$, while the vertical one by $z$; and $c$ is the wave velocity.

As a consequence of the relatively large length of the wire, the wave will be attenuated. Attenuation is the term used to account for loss of wave amplitude due to all mechanisms, including absorption, scattering, and mode conversion.

The model of attenuation is phenomenological, meaning it agrees well in practice but is not easily supported by theory.

The amplitude decay will be modelled as

$$
A(z)=A_{0} e^{-\mu_{A}\left|x-x_{0}\right|}
$$

Where $\mu_{A}$ is called the amplitude attenuation factor and has units $\mathrm{cm}^{-1}$ and $x_{0}$ is the coordinate of the point where the pantograph strikes the contact wire.

Since $20 \log _{10}\left(A(z) / A_{0}\right)$ is the amplitude drop in decibels $(\mathrm{dB})$, it is useful to define the attenuation coefficient $\alpha$ as $\alpha=20 \log _{10}(e) \mu_{A} \sim 8.7 \mu_{A}$.

The absorption coefficient of a material is generally dependent on frequency $f$, and a good model for this dependency is $\alpha=a f^{b}$. The rough approximation that $\mathrm{b}=1$ is often used and, hence, Equation 2a may be written as: 


$$
A(z)=A_{0} e^{-a f x / 8.7}
$$

The boundary conditions are:

$$
z(0, t)=0 \text { and } z(L, t)=0
$$

And the initial conditions are:

$$
z(x, 0)=0 \text { and } \frac{\partial z}{\partial t}(x, 0)=0
$$

Except for $\frac{\partial z}{\partial t}\left(x_{0}, 0\right)=v \delta\left(x-x_{0}\right)$, where $\delta$ is the amplitude Dirac impulse (4) and $v$ the raising velocity of the pantograph [11].

In order to obtain the solution of the wave equation defined by relations (1-3) by means of the Fourier series (5), the equation used for the Dirac impulse will be [12].

$$
\partial(x-x 0)=\frac{2}{L} \sum_{n=1}^{\infty} \sin \frac{n \pi x_{0}}{L} \sin \frac{n \pi x}{L} .
$$

Using the separation of variables method and taking into account the boundary and the initial conditions, the general solution of a vibrating string of length $L$ can be written as the sum of the normal modes (the harmonics):

$$
z(x, t)=\sum_{n=1}^{\infty} A_{n} \sin \omega_{n} t \sin k_{n} x,
$$

Where the pulsation $\omega_{n}$, the wave number $k_{n}$, and the wave length $\lambda_{n}$, fulfil the relations:

$$
\lambda_{n}=\frac{2 L}{n} \quad \lambda_{n} v_{n}=c \quad k_{n}=\frac{2 \pi}{\lambda_{n}} \quad \omega_{n}=2 \pi v_{n} .
$$

According to (6) and (7) the velocity of the vibration is:

$$
\frac{\partial z}{\partial t}=\sum_{n=1}^{\infty} \omega_{n} A_{n} \cos \omega_{n} t \sin \frac{n \pi x}{L} .
$$

Introducing (6) and (7) in the initial condition at the point $x_{0}$, (3), we obtain for the amplitudes the formula:

$$
A_{n}=\frac{2 L v}{\pi n c} \sin \frac{n \pi x_{0}}{L}
$$

And the solution:

$$
z(x, t)=\sum_{n=1}^{\infty} \frac{2 L v}{\pi n c} \sin \frac{n \pi x_{0}}{L} \sin \frac{n \pi c t}{L} \sin \frac{n \pi x}{L}
$$




\section{Waveform and amplitude}

In order to obtain the wave amplitude it should be noticed that in (10) the wavelength of the expansion is $2 L$, while the length of the wire is $L$.

Following a heuristic reasoning it may be figured that, while on the second half of the wire the initial conditions are zero, the terms of (5) may have opposite values, as the functions are even and, respectively, odd, they may be equal on the first half, in order to get the impulse.

Of course, this thing may be rigorously proved. For this reason, the function may be expanded over the first half of the period as in (8). The values of (8) which are outside of this interval have no importance for the solution.

In order to obtain the amplitude of the resulting wave form, the Fourier series decomposition will be studied.

As in the case of the Dirac impulse, it will be considered that the wave is periodic and, more than that, the period is $2 L$, from $-L$ to $L$. We also assume that the function is odd (which means that $A_{n}=0$ ). The Fourier analysis of the oscillation will gives a sine series (because the oscillation is an odd function of time). The coefficients may be easily obtained by replacing $x$ with $x_{0}$ in (9) and they are given by the equation:

$$
B_{n}=\frac{2 L v}{\pi n c} \sin ^{2} \frac{n \pi x}{L}, \quad z\left(x_{0}, t\right)=\sum_{n=0}^{\infty} B_{n} \sin \frac{n \pi c t}{L} .
$$

The most impressive result regarding the waveform equation (10) is that the sum of the harmonics is the Fourier series of a rectangular wave centred in $x_{0}, 2 c t$ wide and $L v / 2 c$ high.

The values in the interval 0 to $L$ will not be affected and the calculus will be simplified. For the above mentioned waveform we obtain a Fourier series were the coefficients are given by (12).

$$
B_{n}=\frac{-2 A}{\pi n}\left(\cos \frac{2 \pi n\left(x_{0}+c t\right)}{2 L}-\cos \frac{2 \pi n\left(x_{0}-c t\right)}{2 L}\right),
$$

Where $A$ is the amplitude of the rectangular wave.

If the difference of cosine in (12) will be transformed in product of sinuses and (10) will be taken into account, the expression of the square wave amplitude (13) is obtained:

$$
A=\frac{L v}{2 c} .
$$

It follows that the wave amplitude is proportional to the distance between the droppers, $L$, with the pantograph strike speed, $v$ and in inverse proportion to the wave speed, $c$. As we mentioned before, if the wire tension increases, the wave speed also increases. Also, the amplitude does not depend on the contact point position.

The result is in good agreement with experimental studies $[13,14]$ and may be used for theoretical studies and design.

\section{Application}

An application example is described in the present section. According to available experimental results, e.g. [6], the accounted wave speed $c$ is $450 \mathrm{~km} / \mathrm{h}$. Further, the assumed pantograph strike speed $v$ is $0.5 \mathrm{~m} / \mathrm{s}$. 
Table 1. Application parameters.

\begin{tabular}{|l|l|l|l|}
\hline Parameter & Value & Description & Units \\
\hline$L$ & 100 & Wire length & $\mathrm{m}$ \\
\hline$c$ & 450 & Wave speed & $\mathrm{km} / \mathrm{h}$ \\
\hline$x_{0}$ & $2 L / 3$ & Pantograph strike point & $\mathrm{m}$ \\
\hline$v$ & 0.5 & Pantograph strike velocity & $\mathrm{m} / \mathrm{s}$ \\
\hline
\end{tabular}

The values of the application parameters are given in Table 1 . The value of the wire length $L$ will further be assumed to be $100 \mathrm{~m}$ and the coordinate of the pantograph strike $x_{0}$, $2 L / 3$.

The amplitude spectrum of the wire transverse wave has a similar structure to the Dirac impulse, equation (9) and, accordingly, has the same characteristics.

The Fourier amplitude spectrum of the wire transverse wave is represented in Figure 1. Every third harmonic is null because the contact point is at $2 / 3$ of the wire length.

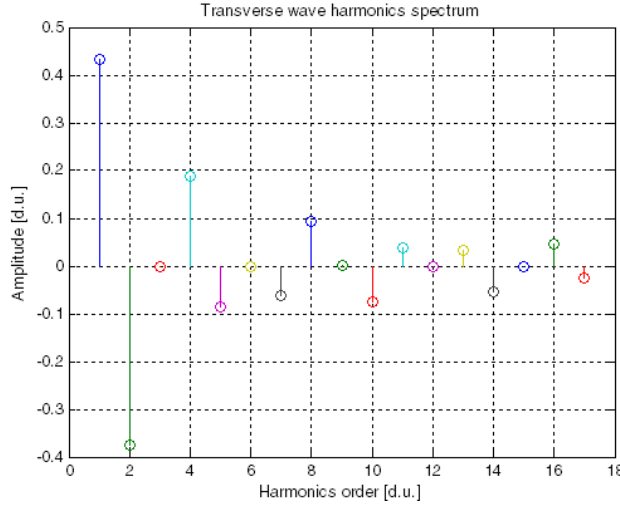

Fig. 1. Transverse wave harmonics spectrum.

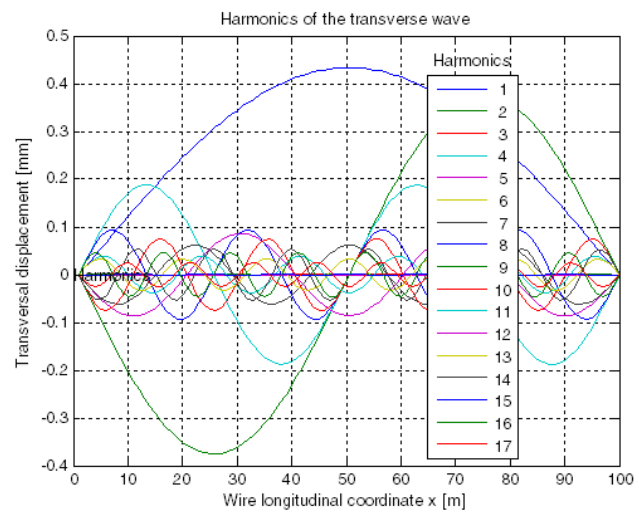

Fig. 2. First $n=17$ harmonics of the transverse wave.

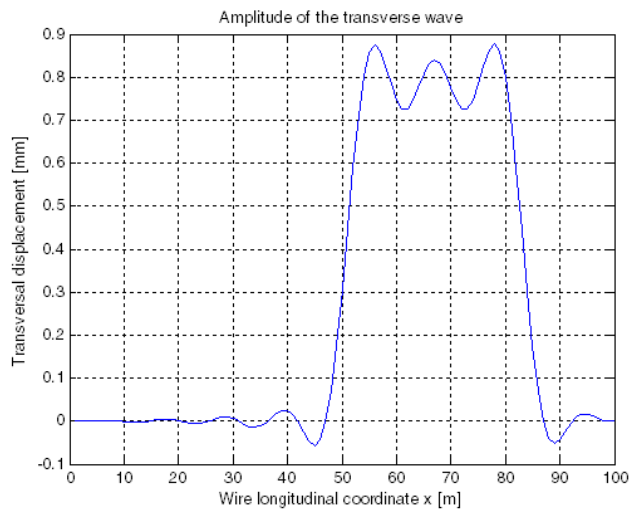

Fig. 3. Transverse wave ( $n=17$ harmonics).

The first harmonics of the wave solution (10) are further represented in Figure 2. The amplitude of each harmonic corresponds to the spectrum plot, Figure 1.

Figure 3 contains a plot of the transverse wave resulted by the superposition of the first $\mathrm{n}=17$ harmonics. The symmetrical evolution of the wave fronts around the contact point can be observed. 


\section{Conclusions}

An analytic solution of the transverse waves generated in a wire by an impulse is obtained in the present work. The impulse is modelled as a Dirac impulse and the solution is obtained using Fourier series.

The wire oscillation has a square waveform; the amplitude is proportional to the wire length and to the strike speed $v$, and in inverse proportion to the wave speed, $c$. The amplitude does not depend on the contact point position.

The present theoretical result is applied to the study of the study of the contact lines oscillations due to electric vehicles pantographs rising. The result is in good agreement with experimental studies.

A better understanding of the phenomena related to the pantograph-wire coupling allows avoiding the occurrence of the electric arch which may cause malfunctioning or may even damage the wire.

\section{References}

1. G. Popa, S. Arsene, M. Mihailescu, International Conference on Electrical Systems for Aircraft, Railway and Ship Propulsion, ESARS, Art. no. 6387408 (2012)

2. G. Popa, S. Arsene, M. Mihailescu, International Conference on Electrical Systems for Aircraft, Railway and Ship Propulsion, ESARS, Art. no. 6387409 (2012)

3. G. Popa, S. Arsene, M. Mihailescu, International Conference on Electrical Systems for Aircraft, Railway and Ship Propulsion, ESARS, Art. no. 6387410 (2012)

4. G. Popa, S. Arsene, International Conference on Electrical Systems for Aircraft, Railway and Ship Propulsion, Art. no. 5665213 (2010)

5. G. Popa, IEEE International Conference on Automation, Quality and Testing, Robotics, Art. no. 4022848, 205-208 (2006)

6. F. Kiesling, R. Puschmann, A. Schmieder, Phil. Trans. Roy. Soc. Siemens Aktiegensellschaft, 439-456 (2001)

7. A. Renger, Kombinat engine fabrication - electrotechnical shop (Henningsdorf, 1987)

8. Y.T. Li, R. Wong, Integral and series representations of the Dirac delta function, Commun. Pure Appl. Anal. 7 (2), 229-247 (2008)

9. F.S. Crawford, Berkeley Physics Course, vol. III (Education Development Center, 1965)

10. R. Buksch, Wiessen-schaftliche Berichte AEG-Telefunken, 52(5), 250-262 (1979)

11. F. Kiesling, M. Semrau, H. Tessun, H. Zweig, Electrische Bahnen, 92(8), 234-240 (1994)

12. R.N. Bracewell, The Fourier Transform and its Applications (McGraw-Hill, 1965, 2nd ed. 1978, revised 1986)

13. J.A. Zagzebski, Essentials of Ultrasound Physics (Mosby Inc., 1996)

14. C. Vera, J. Paulin, B. Suarez, P. Rodriguez, Proceedings of the Eight International Conference on Maintenance \& Renewal of Permanent Way; Power \& Signaling; Structures \& Earthworks (London, U.K., 2005)

15. R. Shingo, A. Mitsuo, Y. Masatsugu, Dynamics \& Design Conference, 1292-1297 (2001) 